CUBO A Mathematical Journal Vol.16, Noㅇ, (55-65). October 2014

\title{
S-asymptotically $\omega$-periodic solution for a nonlinear differential equation with piecewise constant argument in a Banach space
}

\author{
William Dimbour \& Jean-Claude Mado \\ Laboratoire C.E.R.E.G.M.I.A. \\ Université des Antilles et de la Guyane, \\ Campus Fouillole 97159 Pointe-à-Pitre Guadeloupe (FWI) \\ William.Dimbour@univ-ag.fr, Jean-Claude.Mado@univ-ag.fr
}

\begin{abstract}
In this paper, we give some sufficient conditions for the existence and uniqueness of S-asymptotically $\omega$-periodic (mild) solutions for a differential equation with piecewise constant argument, when $\omega$ is an integer. An example is also given in order to illustrate the result.
\end{abstract}

\section{RESUMEN}

En este artículo entregamos algunas condiciones suficientes para la existencia y unicidad de las soluciones mild $\omega$-periódicas S-asintóticas para una ecuación diferencial semilineal con argumento constante por tramos en un espacio de Banach cuando $\omega$ es un entero. Luego, entregamos ejemplos para ilustrar nuestros resultados.

Keywords and Phrases: S-asymptotically $\omega$-periodic function, differential equations with piecewise constant argument, semigroup

2010 AMS Mathematics Subject Classification: 34K05; 34A12; 34A40. 


\section{Introduction}

Let $(\mathbb{X},\|\|$.$) be a Banach space. This work is concerned with the existence of S-asymptotically$ $\omega$-periodic solutions to the differential equations with piecewise constant argument of the form

$$
\text { (2) }\left\{\begin{array}{l}
x^{\prime}(t)=A x(t)+A_{0} x([t])+g(t, x(t)) \\
x(0)=c_{0}
\end{array}\right.
$$

where $\mathrm{A}$ is the infitesimal generator of an exponentially stable $\mathrm{C}_{0}$-semigroup acting on $\mathbb{X}$, [.] is the largest interger function and $g: \mathbb{R}^{+} \times \mathbb{X} \rightarrow \mathbb{X}$ is an appropriate function that will be defined later.

They are a some papers dealing wiht S-asymptotically $\omega$-periodic functions. Qualitative properties of such functions are discussed for instance in [1] and [5. In [5], a new composition theorem for such functions is also presented. In [7, Lizama and N'Guérékata created a chart establishing a general relationship between S-asymptotically $\omega$-periodic functions and various subspaces of $B C(\mathbb{R}, \mathbb{X})$. [1], 3], 4], [5], 6], [7] study the existence of S-asymptotically $\omega$-periodic solutions of diffenretial equations in finite as well infinite dimensional spaces.

They are also some papers dealing with the existence of almost automorphic solutions for differential equation with piecewise constant argument. In [8], Nguyen Van Minh and Tran Tat Dat give sufficient spectral conditions for the almost automorphy of bounded solutions to differential equations wtih piecewise constant argument of the form

$$
x^{\prime}(t)=A x(t)+f(t), t \in \mathbb{R},
$$

where $A$ is a bounded linear operator in $\mathbb{X}$ and $f$ in an $\mathbb{X}$-valued almost automorphic function. In 2, Dimbour generalize the work of Nguyen Van Minh and Tran Tat Dat, giving also sufficient spectral conditions for the almost automorphy of bounded solutions to differential equations wtih piecewise constant argument of the form

$$
x^{\prime}(t)=A(t) x(t)+f(t), t \in \mathbb{R},
$$

where $A(t)$ is an almost automorphy operator and $f$ in an $\mathbb{X}$-valued almost automorphic function. Following this work, we study in this paper S-asymptotically $\omega$-periodic solutions of (2). We first study the linear system associated to (2). Then using the Banach's theorem, we showed the existence of S-asymptotically $\omega$-periodic solutions for the following equation

$$
\left\{\begin{array}{l}
x^{\prime}(t)=A x(t)+A_{0} x([t])+f(t) \\
x(0)=c_{0}
\end{array}\right.
$$

The rest of the paper is organise as follows. In section 2, we recall some results on S-asymptotically $\boldsymbol{\omega}$-periodic functions. In section 3 , first of all considering the $\boldsymbol{c}_{0}$ semigroup theory ([9]), we define a mild solution of (1). We give some sufficient conditions for the existence and uniqueness of S-asymptotically $\omega$-periodic solutions of (1) and (2). These results are obtained by mean of the Banach fixed point principle, when $\omega$ is an integer. In the section 4, we give an example. 


\section{PRELIMINARIES}

Let $\mathbb{X}$ be a Banach space. $\mathrm{BC}\left(\mathbb{R}^{+}, \mathbb{X}\right)$ denotes the space of the continuous bounded functions from $\mathbb{R}^{+}$into $\mathbb{X}$; endowed with the norm $\|f\|_{\infty}:=\sup _{t} \geq 0\|f(t)\|$, it is a Banach space. $C_{0}\left(\mathbb{R}^{+}, \mathbb{X}\right)$ denotes the space of the continuous functions from $\mathbb{R}$ into $\mathbb{X}$ such that $\lim _{t \rightarrow \infty} f(t)=0$; it is a Banach subspace of $B C\left(\mathbb{R}^{+}, \mathbb{X}\right)$. When we fix a positive number $\omega, P_{\omega}(\mathbb{X})$ denotes the space of all continuous $\omega$-periodic functions from $\mathbb{R}^{+}$into $\mathbb{X}$; it is a Banach subspace of $\mathrm{BC}\left(\mathbb{R}^{+}, \mathbb{X}\right)$ under the sup norm.

Definition 1. Let $\mathrm{f} \in \mathrm{BC}\left(\mathbb{R}^{+}, \mathbb{X}\right)$ and $\omega>0$. We say that $\mathrm{f}$ is asymptotically $\boldsymbol{\omega}$-periodic if $\mathrm{f}=\mathrm{g}+\mathrm{h}$ where $\mathrm{g} \in \mathrm{P}_{\omega}(\mathbb{X})$ and $\mathrm{h} \in \mathrm{C}_{0}\left(\mathbb{R}^{+}, \mathbb{X}\right)$.

We denote by $\operatorname{AP}(\mathbb{X})$ the set of all asymptotically $\omega$-periodic functions from $\mathbb{R}^{+}$to $\mathbb{X}$. It is a Banach space under the sup norm.

Definition 2. A function $\mathrm{f} \in \mathrm{BC}\left(\mathbb{R}^{+}, \mathbb{X}\right)$ is called S-asymptotically $\omega$-periodic if there exists $\omega$ such that $\lim _{\mathrm{t} \rightarrow \infty}(\mathrm{f}(\mathrm{t}+\mathrm{\omega})-\mathrm{f}(\mathrm{t}))=0$. In this case we say that $\omega$ is an asymptotic period of $f$ and that $f$ is $S$-asymptotically $\omega$-periodic.

We will denote by $\operatorname{SAP}_{\omega}(\mathbb{X})$, the set of all S-asymptotically $\omega$-periodic functions from $\mathbb{R}^{+}$to $\mathbb{X}$. Then we have

$$
\operatorname{AP}_{\omega}(\mathbb{X}) \subset \operatorname{SAP}_{\omega}(\mathbb{X})
$$

The inclusion is strict. Indeed consider the function $f: \mathbb{R}^{+} \rightarrow c_{0}$ where $c_{0}=\left\{x=\left(x_{n}\right)_{n \in \mathbb{N}}\right.$ : $\left.\lim _{n \rightarrow \infty} x_{n}=0\right\}$ equipped with the norm $\|x\|=\sup _{n \in \mathbb{N}}|x(n)|$, and $\left(f(t)=\frac{2 n t^{2}}{t^{2}+n^{2}}\right)_{n \in \mathbb{N}}$. Then $\mathrm{f} \in \mathrm{SAP}_{\boldsymbol{\omega}}(\mathbb{X})$ but $\mathrm{f} \notin A \mathrm{P}_{\omega}(\mathbb{X})$ (see [5] Example 3.1).

The following result is due to Henriquez-Pierri-Tàboas; Proposition 3.5 in [5].

Theorem 1. Endowed with the norm $\|\cdot\|_{\infty}, \operatorname{SAP}_{\omega}(\mathbb{X})$ is a Banach space.

Corollary 1. (see [1], Corollary 3.10 p.5) Let $\mathbb{X}$ and $\mathbb{Y}$ be two Banach spaces, and let $A \in$ $\mathcal{L}(\mathbb{X}, \mathbb{Y})$. Then when $f \in \operatorname{SAP}_{\omega}(\mathbb{X})$, we have Af $:=[t \rightarrow \operatorname{Af}(t)] \in \operatorname{SAP}_{\omega}(\mathbb{Y})$.

For the sequel we consider asymptotically $\omega$-periodic functions with parameters.

Definition 3. (see [5]) A continuous function $\mathrm{g}:[0, \infty[\times \mathbb{X} \rightarrow \mathbb{X}$ is said to be uniformly $S$ asymptotically $\omega$-periodic on bounded sets if for every bounded set $\mathrm{K} \subset \mathbb{X}$, the $\operatorname{set}\{\mathrm{f}(\mathrm{t}, \mathrm{x}): \mathrm{t} \geq$ $0, x \in \mathrm{K}\}$ is bounded and $\lim _{\mathrm{t} \rightarrow \infty}(\mathrm{f}(\mathrm{t}, \mathrm{x})-\mathrm{f}(\mathrm{t}+\omega, \mathrm{x}))=0$ uniformly on $\mathrm{x} \in \mathrm{K}$.

Definition 4. (see [5]) A continuous function $\mathrm{g}:[0, \infty[\times \mathbb{X} \rightarrow \mathbb{X}$ is said to be asymptotically uniformly continuous on bounded sets if for every $\epsilon>0$ and every bounded set $\mathrm{K} \subset \mathbb{X}$, there exist $\mathrm{L}_{\epsilon, \mathrm{K}}>0$ and $\delta_{\epsilon, \mathrm{K}}>0$ such that $\|\mathrm{f}(\mathrm{t}, \mathrm{x})-\mathrm{f}(\mathrm{t}, \mathrm{y})\|<\epsilon$ for all $\mathrm{t} \geq \mathrm{L}_{\epsilon, \mathrm{K}}$ and all $\mathrm{x}, \mathrm{y} \in \mathrm{K}$ with $\|x-y\|<\delta_{\epsilon, \mathrm{K}}$. 
Theorem 2. (see [5]) Let $\mathrm{g}:[0, \infty[\times \mathbb{X} \rightarrow \mathbb{X}$ be a function which uniformly S-asymptotically $\omega$-periodic on bounded sets and asymptotically uniformly continuous on bounded sets. Let $\mathfrak{u}$ : $[0, \infty[\rightarrow \mathbb{X}$ be S-asymptotically $\omega$-periodic function. Then the Nemytskii function $\phi():.=\mathrm{f}(., \mathrm{u}())$. is $S$-asymptotically $\omega$-periodic function.

\section{Main result}

\subsection{The linear case}

Definition 5. A solution of Eq.(1) on $\mathbb{R}^{+}$is a function $x(t)$ that satisfies the conditions:

$1-x(t)$ is continuous on $\mathbb{R}^{+}$.

2-The derivative $x^{\prime}(t)$ exists at each point $t \in \mathbb{R}^{+}$, with possible exception of the points $[t] \in \mathbb{R}^{+}$ where one-sided derivatives exists.

3-Eq.(1) is satisfied on each interval $[\mathrm{n}, \mathrm{n}+1[$ with $\mathrm{n} \in \mathbb{N}$.

Let $T(t)$ be the $C_{0}$ semigroup generated by $A$ and $x$ a solution of (1). We assume that $f \in L^{1}\left(\mathbb{R}^{+}, \mathbb{X}\right)$. Then the function $g$ defined by $g(s)=T(t-s) x(s)$ is differentiable for $s<t$.

$$
\begin{gathered}
\frac{d g(s)}{d s}=-A T(t-s) x(s)+T(t-s) x^{\prime}(s) \\
=-A T(t-s) x(s)+T(t-s) A x(s)+T(t-s) A_{0} x([s])+T(t-s) f(s) \\
(3)=T(t-s) A_{0} x([s])+T(t-s) f(s)
\end{gathered}
$$

Since $f \in L^{1}(\mathbb{R}, \mathbb{X}), T(t-s) f(s)$ is integrable on $[0, t]$ with $t \in \mathbb{R}^{+}$. The function $x([s])$ is a step function. Therefore $x([s])$ is integrable on $[0, t]$ with $t \in \mathbb{R}^{+}$. Integrating (3) on $[0, t]$, we obtain that

$$
x(t)-T(t) x(0)=\int_{0}^{t} T(t-s) A_{0} x([s]) d s+\int_{0}^{t} T(t-s) f(s) d s .
$$

Therefore, we define

Definition 6. Let $\mathrm{T}(\mathrm{t})$ be the $\mathrm{C}_{0}$ semigroup generated by $\mathrm{A}$ and $\mathrm{f} \in \mathrm{L}^{1}\left(\mathbb{R}^{+}, \mathbb{X}\right)$. The function $x \in \mathcal{C}\left(\mathbb{R}^{+}, \mathbb{X}\right)$ given by

$$
x(t)=T(t) c_{0}+\int_{0}^{t} T(t-s) A_{0} x([s]) d s+\int_{0}^{t} T(t-s) f(s) d s
$$

is the mild solution of the equation (1).

Now we make the following hypothesis. 
(H.1) The operator A is the infinitesimal generator of an exponentially stable semigroup $(T(t))_{t \geq 0}$ such that there exist constants $M>0$ and $\delta>0$ with

$$
\|\mathrm{T}(\mathrm{t})\|_{\mathrm{B}(\mathrm{X})} \leq M e^{-\delta \mathrm{t}}, \forall \mathrm{t} \geq 0 .
$$

Lemma 1. We assume that the hypothesis (H.1) is satisfied. Then the function $\mathrm{L}$ defined by

$$
\mathrm{L}(\mathrm{t})=\mathrm{T}(\mathrm{t}) \times(0)
$$

belongs to $\operatorname{SAP}_{\omega}(\mathbb{X})$.

Proof.

$$
\begin{aligned}
\|\mathrm{L}(\mathrm{t}+\omega)-\mathrm{L}(\mathrm{t})\| & =\|\mathrm{T}(\mathrm{t}+\omega) x(0)-\mathrm{T}(\mathrm{t}) x(0)\| \\
& \leq\|\mathrm{T}(\mathrm{t}+\omega) x(0)\|+\|\mathrm{T}(\mathrm{t}) x(0)\| \\
\leq & M e^{-\delta(t+\omega)}+\mathrm{Me}^{-\delta \mathrm{t}}
\end{aligned}
$$

Since $\delta>0$, we deduce that

$$
\lim _{t \rightarrow \infty}\|\mathrm{L}(\mathrm{t}+\omega)-\mathrm{L}(\mathrm{t})\|=0 .
$$

Then $\mathrm{L} \in \operatorname{SAP}_{\omega}(\mathbb{X}) . \square$

Lemma 2. We assume that the hypothesis (H.1) is satisfied. We assume also that $\mathrm{A}_{0}$ is a linear bounded operator and $\omega \in \mathbb{N}$. We define the nonlinear operator $\wedge_{1}$ by: for each $\phi \in \operatorname{SAP}_{\omega}(\mathbb{X})$

$$
\left(\wedge_{1} \phi\right)(t)=\int_{0}^{t} T(t-s) A_{0} \phi([s]) d s .
$$

Then the operator $\wedge_{1}$ maps $\operatorname{SAP}_{\omega}(\mathbb{X})$ into itself.

Proof. We put $v(t)=\int_{0}^{t} T(t-s) A_{0} \phi([s]) d s$. For $t \geq 0$, we have

$$
\begin{gathered}
v(t+\omega)-v(t)=\int_{0}^{t+\omega} T(t+\omega-s) A_{0} \phi([s]) d s-\int_{0}^{t} T(t-s) A_{0} \phi([s]) d s \\
=\int_{0}^{\omega} T(t+\omega-s) A_{0} \phi([s]) d s+\int_{\omega}^{t+\omega} T(t+\omega-s) A_{0} \phi([s]) d s \\
\quad-\int_{0}^{t} T(t-s) A_{0} \phi(s) d s .
\end{gathered}
$$

Then we have

$$
\|v(t+w)-v(t)\| \leq\left\|I_{1}(t)\right\|+\left\|I_{2}(t)\right\|
$$

where

$$
\mathrm{I}_{1}(\mathrm{t})=\int_{0}^{\omega} \mathrm{T}(\mathrm{t}+\omega-\mathrm{s}) \mathrm{A}_{0} \phi([s]) \mathrm{ds}
$$


and

$$
I_{2}(t)=\int_{\omega}^{t+\omega} T(t+\omega-s) A_{0} \phi([s]) d s-\int_{0}^{t} T(t-s) A_{0} \phi([s]) d s .
$$

Observing that

$$
\mathrm{I}_{1}(\mathrm{t})=\mathrm{T}(\mathrm{t}) \int_{0}^{\omega} \mathrm{T}(\omega-\mathrm{s}) A_{0} \phi([s]) \mathrm{ds}
$$

and using the fact that $(\mathrm{T}(\mathrm{t}))_{\mathrm{t} \geq 0}$ is exponentially stable, we deduce that

$$
\left\|\mathrm{I}_{1}(\mathrm{t})\right\| \leq M e^{-\delta \mathrm{t}}\|v(\omega)\|
$$

Therefore $\lim _{t \rightarrow \infty} I_{1}(t)=0$.

Now, show that $\lim _{t \rightarrow \infty}\|\phi([t+\omega])-\phi([t])\|=0$.

We have that $\lim _{t \rightarrow \infty}\|\phi(t+\omega)-\phi(t)\|=0$.

Therefore:

$$
\forall \epsilon>0, \exists \mathrm{T}_{\epsilon}^{0} \in \mathbb{R}^{+}, \forall \mathrm{t}>\mathrm{T}_{\epsilon}^{0} \Rightarrow\|\phi(\mathrm{t}+\omega)-\phi(\mathrm{t})\|<\epsilon .
$$

We put $T_{\epsilon}=\left[T_{\epsilon}^{0}\right]+1$. Let $\epsilon>0$. For $t>T_{\epsilon}$, we observe that $[t] \geq T_{\epsilon}$ because $T_{\epsilon}$ is an integer. We deduce so that

$$
\forall \epsilon>0, \exists \mathrm{T}_{\epsilon} \in \mathbb{R}^{+}, \forall \mathrm{t}>\mathrm{T}_{\epsilon} \Rightarrow\|\phi([\mathrm{t}]+\omega)-\phi([\mathrm{t}])\|<\epsilon .
$$

Since $\omega$ is an integer, we observe that

$$
\forall \epsilon>0, \exists \mathrm{T}_{\epsilon} \in \mathbb{R}^{+}, \forall \mathrm{t}>\mathrm{T}_{\epsilon} \Rightarrow\|\phi([\mathrm{t}+\omega])-\phi([\mathrm{t}])\|<\epsilon .
$$

Let $\epsilon>0$, we can find $T_{\epsilon}$ sufficiently large such that

$$
\|\phi([t+\omega])-\phi([t])\|<\frac{\delta}{M\left\|A_{0}\right\|} \epsilon, \text { for } t>T_{\epsilon} .
$$

Let's write

$$
\left.\mathrm{I}_{2}(\mathrm{t})=\int_{0}^{\mathrm{t}} \mathrm{T}(\mathrm{t}-\mathrm{s})\right) A_{0}(\phi([s+\omega])-\phi([s])) \mathrm{d} s
$$

then we obtain

$$
\left.\left.\left\|I_{2}(t)\right\| \leq \| \int_{0}^{T_{\epsilon}} T(t-s)\right) A_{0}(\phi([s+\omega])-x([s])) d s\|+\| \int_{T_{\epsilon}}^{t} T(t-s)\right) A_{0}(x([s+\omega])-\chi([s])) d s \| .
$$

Observing that

$$
\begin{aligned}
\left\|\int_{0}^{T_{\epsilon}} \mathrm{T}(\mathrm{t}-\mathrm{s}) \mathrm{A}_{0}(\phi([s+\omega])-\phi([s])) \mathrm{d} \boldsymbol{s}\right\| & \leq \int_{0}^{\mathrm{T}_{\epsilon}}\|\mathrm{T}(\mathrm{t}-\mathrm{s})\|\left\|A_{0}\right\|\|\phi([s+\omega])-\phi([\mathrm{s}])\| \\
& \leq \int_{0}^{\mathrm{T}_{\epsilon}} M e^{-\delta(\mathrm{t}-\mathrm{s})}\left\|\mathrm{A}_{0}\right\| 2\|\phi\|_{\infty} \mathrm{d} s
\end{aligned}
$$




$$
\leq \frac{M\left\|A_{0}\right\| 2\|\phi\|_{\infty}}{\delta}\left(e^{-\delta\left(t-T_{e}\right)}-e^{-\delta t}\right)
$$

we deduce that

$$
\lim _{t \rightarrow \infty} \int_{0}^{T_{\epsilon}} \mathrm{T}(\mathrm{t}-\mathrm{s})(\Phi([s+\omega])-\Phi([s])) \mathrm{d} s=0 .
$$

We have also that

$$
\begin{aligned}
\left\|\int_{T_{\epsilon}}^{t} T(t-s) A_{0}(\Phi([s+\omega])-\Phi([s])) d s\right\| & \leq \int_{T_{\epsilon}}^{t} M e^{-\delta(t-s)}\left\|A_{\mathcal{O}}\right\| \frac{\delta}{M\left\|A_{\mathcal{O}}\right\|} \epsilon d s \\
& \leq \epsilon \int_{T_{\epsilon}}^{t} \delta e^{-\delta(t-s)} \mathrm{ds} \\
& \leq \epsilon\left(1-e^{-\delta\left(t-T_{\epsilon}\right)}\right) \\
& \leq \epsilon
\end{aligned}
$$

Therefore $\lim _{t \rightarrow \infty} \int_{T_{e}}^{t} T(t-s) A_{0}(\Phi([s+w])-\Phi([s])) d s=0$.

We deduce so that $\lim _{t \rightarrow \infty} I_{2}(t)=0$, this proves that $\wedge_{1} \in \operatorname{SAP}_{\omega}(\mathbb{X})$.

Theorem 3. We assume that the hypothesis (H.1) is satisfied. Let $\omega \in \mathbb{N}$. We assume also that $\mathrm{f}$ is a $S$ asymptotically $\omega$-periodic function. Then the equation (1) has a unique $S$ asymptotically $\omega$-periodic solution if

$$
\Theta:=\frac{M}{\delta}\left\|A_{0}\right\|<1
$$

Proof. Define the nonlinear operator $\Gamma: \operatorname{SAP}_{\omega}(\mathbb{X}) \mapsto \operatorname{SAP}_{\omega}(\mathbb{X})$

$$
(\Gamma \mathrm{u})(\mathrm{t}):=\mathrm{L}(\mathrm{t})+\left(\wedge_{1} \mathrm{u}\right)(\mathrm{t})+\wedge_{2}(\mathrm{t})
$$

for every $u \in \operatorname{SAP}_{\omega}(\mathbb{X})$, where

$$
\left(\wedge_{1} u\right)(t)=\int_{0}^{t} T(t-s) A_{0} \phi([s]) d s
$$

and

$$
\wedge_{2}(t)=\int_{0}^{t} T(t-s) f(s) d s .
$$

We satisfy that the nonlinear operator $\Gamma$ is well defined.

The lemma 1 show that $\mathrm{L}(\mathrm{t})$ is is a $\mathrm{S}$ asymptotically $\boldsymbol{\omega}$-periodic. The lemma 2 show that the operator $\wedge_{1}$ maps $\operatorname{SAP}_{\omega}(\mathbb{X})$ into itself. Then the nonlinear operator $\Gamma$ maps $\operatorname{SAP}_{\omega}(\mathbb{X})$ into itself. Since $\|\mathrm{L}(\mathrm{t})\| \leq M e^{-\delta t}, \forall \mathrm{t} \geq 0$, we observe that $\mathrm{L}(\mathrm{t}) \in \mathrm{C}_{0}\left(\mathbb{R}^{+}, \mathbb{X}\right)$.

For every $\phi, \psi \in \operatorname{SAP}_{\omega}(\mathbb{X})$

$\|\Gamma(\phi)(\mathrm{t})-\Gamma(\psi)(\mathrm{t})\|$ 


$$
\begin{aligned}
& =\| T(t) c_{0}+\int_{0}^{t} T(t-s) A_{0} \phi([s]) d s+\int_{0}^{t} T(t-s) f(s) d s \\
& -T(t) c_{0}-\int_{0}^{t} T(t-s) A_{0} \psi([s]) d s-\int_{0}^{t} T(t-s) f(s) d s \| \\
& \leq\left\|\int_{0}^{t} T(t-s) A_{0}(\phi([s])-\psi([s])) d s\right\| \\
& \leq \int_{0}^{t}\|T(t-s)\|\left\|A_{0}\right\|\|\phi([s])-\psi([s])\| d s \\
& \leq \int_{0}^{t}\|T(t-s)\|\left\|A_{0}\right\|\|\phi-\psi\|_{\infty} d s \\
& \leq \int_{0}^{t} M e^{-\delta(t-s)} d s\left\|A_{0}\right\|\|\phi-\psi\|_{\infty} \\
& \quad \leq \frac{M}{\delta}\left\|A_{0}\right\|\|\phi-\psi\|_{\infty} .
\end{aligned}
$$

Therefore, if $\Theta<1$, then the equation (1) has a unique S asymptotically $\omega$-periodic solution.

\subsection{The nonlinear case}

Definition 7. A solution of Eq.(2) on $\mathbb{R}^{+}$is a function $x(t)$ that satisfies the conditions:

$1-x(t)$ is continuous on $\mathbb{R}^{+}$.

2-The derivative $x^{\prime}(\mathrm{t})$ exists at each point $\mathrm{t} \in \mathbb{R}^{+}$, with possible exception of the points $[\mathrm{t}] \in \mathbb{R}^{+}$ where one-sided derivatives exists.

3-Eq.(2) is satisfied on each interval $[\mathrm{n}, \mathrm{n}+1[$ with $\mathrm{n} \in \mathbb{N}$.

We now make the following assumption.

(H.2) The function $g: \mathbb{R}^{+} \times \mathbb{X} \rightarrow \mathbb{X},(t, u) \rightarrow g(t, u)$ is uniformly S-asymptotically $\omega$-periodic on bounded sets and asymptotically uniformly continuous on bounded sets. There exist constant $\mathrm{K}_{\mathrm{g}} \geq 0$ such that

$$
\|g(t, u)-g(t, v)\| \leq K_{g}\|u-v\|
$$

for all $\mathrm{t} \in \mathbb{R}^{+}$, and $\forall \mathrm{u}, v \in \mathbb{X}$.

Definition 8. Let $\mathrm{T}(\mathrm{t})$ be the $\mathrm{C}_{0}$ semigroup generated by $\mathrm{A}$. The function $\mathrm{x} \in \mathcal{C}\left(\mathbb{R}^{+}, \mathbb{X}\right)$ given by

$$
x(t)=T(t) c_{0}+\int_{0}^{t} T(t-s) A_{0} x([s]) d s+\int_{0}^{t} T(t-s) g(s, x(s)) d s
$$

is the mild solution of the equation (2). 
Theorem 4. We assume that the hypothesis (H.1) and (H.2) are satisfied. Let $\omega \in \mathbb{N}$. Then the equation (2) has a unique $S$ asymptotically $\omega$-periodic solution if

$$
\Theta:=\frac{M}{\delta}\left(\left\|A_{0}\right\|+K_{g}\right)<1 .
$$

Proof. Define the nonlinear operator $\Gamma: \operatorname{SAP}_{\omega}(\mathbb{X}) \mapsto \operatorname{SAP}_{\omega}(\mathbb{X})$

$$
(\Gamma \mathrm{u})(\mathrm{t}):=\mathrm{L}(\mathrm{t})+\left(\wedge_{1} \mathrm{u}\right)(\mathrm{t})+\wedge_{2}(\mathrm{t})
$$

for every $u \in \operatorname{SAP}_{\omega}(\mathbb{X})$, where

$$
\left(\wedge_{1} u\right)(t)=\int_{0}^{t} T(t-s) A_{0} \phi([s]) d s
$$

and

$$
\wedge_{2}(t)=\int_{0}^{t} T(t-s) g(s, x(s)) d s .
$$

Since the hypothesis (H.2) is satisfied, the nonlinear operator $\Gamma$ is well defined.

For every $\phi, \psi \in \operatorname{SAP}_{\omega}(\mathbb{X})$

$\|\Gamma(\phi)(t)-\Gamma(\psi)(t)\|$

$$
\begin{aligned}
& =\| \mathrm{T}(\mathrm{t}) \mathrm{c}_{0}+\int_{0}^{\mathrm{t}} \mathrm{T}(\mathrm{t}-\mathrm{s}) \mathrm{A}_{0} \phi([\mathrm{s}]) \mathrm{d} s+\int_{0}^{\mathrm{t}} \mathrm{T}(\mathrm{t}-\mathrm{s}) \mathrm{g}(\mathrm{s}, \phi(\mathrm{s})) \mathrm{d} s \\
& -T(t) c_{0}-\int_{0}^{t} T(t-s) A_{0} \psi([s]) d s-\int_{0}^{t} T(t-s) g(s, \psi(s)) d s \| \\
& \leq\left\|\int_{0}^{t} \mathrm{~T}(\mathrm{t}-\mathrm{s}) \mathrm{A}_{0}(\phi([s])-\psi([s])) \mathrm{ds}\right\|+\| \int_{0}^{\mathrm{t}} \mathrm{T}(\mathrm{t}-\mathrm{s})(\mathrm{g}(\mathrm{s}, \phi(\mathrm{s}))-\mathrm{g}(\mathrm{s}, \psi(\mathrm{s}))) \mathrm{ds} \\
& \leq \int_{0}^{t}\|\mathrm{~T}(\mathrm{t}-\mathrm{s})\|\left\|\mathrm{A}_{0}\right\|\|\phi([\mathrm{s}])-\psi([\mathrm{s}])\| \mathrm{d} s+\int_{0}^{\mathrm{t}}\|\mathrm{T}(\mathrm{t}-\mathrm{s})\|\|\mathrm{g}(\mathrm{s}, \phi(\mathrm{s}))-\mathrm{g}(\mathrm{s}, \psi(\mathrm{s}))\| \mathrm{d} \mathrm{s} \\
& \leq \int_{0}^{t}\|\mathrm{~T}(\mathrm{t}-\mathrm{s})\|\left\|\mathrm{A}_{0}\right\|\|\phi-\psi\|_{\infty} \mathrm{ds}+\int_{0}^{\mathrm{t}}\|\mathrm{T}(\mathrm{t}-\mathrm{s})\| \mathrm{K}_{\mathrm{g}}\|\phi-\psi\|_{\infty} \mathrm{d} \mathrm{s} \\
& \leq \int_{0}^{t} M e^{-\delta(t-s)} \mathrm{d} s\left\|A_{0}\right\|\|\phi-\psi\|_{\infty}+\int_{0}^{t} M K_{g} e^{-\delta(t-s)} \mathrm{d} s\|\phi-\psi\|_{\infty} \\
& \leq \frac{M}{\delta}\left\|A_{0}\right\|\|\phi-\psi\|_{\infty}+\frac{M}{\delta} K_{g}\|\phi-\psi\|_{\infty} \\
& \leq \frac{M}{\delta}\left(\left\|A_{0}\right\|+K_{g}\right)\|\phi-\psi\|_{\infty}
\end{aligned}
$$

Therefore, if $\Theta<1$, then the equation (2) has a unique S asymptotically $\omega$-periodic solution. 


\section{Application}

As an application, we consider

$$
\left\{\begin{aligned}
\frac{\partial u}{\partial t}(t, x) & =\frac{\partial^{2} u}{\partial x^{2}}(t, x)+\alpha u([t], x)+g(t, u(t, x)) \quad t \in \mathbb{R}^{+}, x \in[0, \pi], \alpha \in \mathbb{R} \\
u(t, 0) & =u(t, \pi)=0 \quad t \in \mathbb{R}^{+} \\
u(0) & =c_{0} \in \mathbb{X}
\end{aligned}\right.
$$

We assume that $(\mathbb{X},\|\cdot\|)=\left(\mathrm{L}^{2}(0, \pi),\|\cdot\|_{2}\right)$ and define

$$
\begin{aligned}
& \mathrm{D}(\mathrm{A})=\left\{\mathrm{u} \in \mathrm{L}^{2}[0, \pi], u(0)=u(\pi)=0\right\} \\
& \mathrm{Au}(\cdot)=\triangle \mathrm{u}=\mathrm{u}^{\prime \prime}(\cdot), \quad \forall \mathrm{u}(\cdot) \in \mathrm{D}(\mathrm{A}) .
\end{aligned}
$$

A is the infinetesimal generator of a semigroup $T(t)$ on $\mathrm{L}^{2}[0, \pi]$ with $\|T(t)\| \leq e^{-t}$ for $t \geq 0$.

Put $u(t)=u(t, \cdot)$ that is $u(t) x=u(t, x),(t, x) \in \mathbb{R}^{+} \times(0, \pi)$. Considering $A_{0}: L^{2}[0, \pi] \mapsto$ $\mathrm{L}^{2}[0, \pi], y \rightarrow \alpha y$, we observe that $A_{0}$ is a linear bounded operator such that $\left\|A_{0}\right\|=|\alpha|$ and $A_{0} u([t])=\alpha u([t], \cdot)$.

Theorem 5. We assume that $\omega \in \mathbb{N}$. Then the system (4) has a unique mild solution S asymptotically $\omega$-periodic if $|\alpha|<1$.

Proof. We have $M=1, \delta=1,\left\|A_{0}\right\|=|\alpha|$. Then we apply the theorem (44) for the system (4).

Received: April 2014. Accepted: May 2014.

\section{References}

[1] J.Blot, P.Cieutat, G.N'Guérékata S-asymptotically $\omega$-periodic functions and applications to evolution equations Afr. diaspora. J.Math. 12 (2011), 113-121.

[2] W. Dimbour, Almost automorphic solutions for a differential equations with piecewise constant argument in a Banach space, Nonlinear Analysis, Vol.74 (2011) 2351-2357.

[3] W. Dimbour, G. N'Guérékata, S-asymptotically $\omega$-periodic solutions to some classes of partial evolution equation, Applied Mathematics and Computation, Vol.218 (2012), 7622-7628.

[4] W. Dimbour, G. Mophou, G. N'Guérékata, S-asymptotically w-periodic solutions for partial differential equations with finite delay, Electronic Journal of Differential Equation, Vol.2011 (2011), 1-12. 
[5] H.R.Henríquez, M.Pierre, P.Táboas On S-asymptotically w-periodic function on Banach spaces and applications, J.Math.Anal.Appl 343(2008), 1119-1130.

[6] H.R.Henríquez, M.Pierre, P.Táboas Existence of S-asymptotically w-periodic solutions for abstract neutral equations, Bull.Austr.Math.Soc 78(2008), 365-382.

[7] C.Lizama, G.N'Guérékata Bounded mild solutions for semilinear integrodifferential equations in Banach space, Integr.Eq.Oper. Theory 68(2010) 207-227.

[8] N.Van Minh, T. Tat Dat, On the Almost Automorphy of bounded solutions of differential equations with piecewise constant argument, Journal of Mathematical analysis and appliction 326(2007), 165-178.

[9] K.Yosida, Functional Analysis, Springer-Verlag (1968). 